Entropy, 2006, 8, 175-181

Full paper

\title{
Generalized Ideal Gas Equations for Structureful Universe
}

\author{
Shahid N. Afridi ${ }^{1}$ and Khalid Khan ${ }^{2}$
}

Department of Physics, Quaid-i-Azam University, Islamabad 45320, Pakistan.

${ }^{1}$ E-mail: snafridi@phys.qau.edu.pk

2 E-mail: mkk@qau.edu.pk

Received: 19 May 2006/ Accepted: 4 September 2006 / Published: 4 September 2006

\begin{abstract}
We have derived generalized ideal gas equations for a structureful universe consisting of all forms of matters. We have assumed a universe that contains superclusters. Superclusters are then made of clusters. Each cluster can be further divided into smaller ones and so on. We have derived an expression for the entropy of such a universe. Our model is rather independent of the geometry of the intermediate clusters. Our calculations are valid for a non-interacting universe within non-relativistic limits. We suggest that structure formation can reduce the expansion rate of the universe.
\end{abstract}

Keywords: set theory; cluster; temperature; pressure; entropy; thermal energy; cosmology. 


\section{Introduction}

Ideal gas equations can be applied to non-interacting dilute gases. Molecules and atoms in gas phase are so far away from each other, it makes a little difference if we ignore the molecular interaction. These molecules and atoms are further subject to Heisenberg uncertainty principle [1]. When dealing with the universe as a whole, we see that the molecules, nucleons and other subatomic particles are not uniformly distributed over the space. There exists various scales in the universe. The nucleons are particles in a star and at a larger scale the stars can be treated as particles of a galaxy and so on. In order to incorporate all scales one is required to generalize the ideal gas equation. In this paper, we have derived generalized gas equations for such a universe. In an earlier paper, based on configuration space, we addressed future orientation of time with the growth of entropy [2]. In the present paper we have extended the same model to phase space. In this model we assume that the universe is structureful which consists of clusters. Each cluster contains subclusters. These subclusters can further be divided into even smaller ones and so on. Such a description is called the universal self-similarity [2]. It should be noted that, for instance, cluster of galaxies are bound, virialized, high over density system, held together by the cluster self gravity [3]. In this perspective we can say that our model intrinsically incorporates gravity.

\section{Model}

We can assume that on large scale the universe is homogeneous, isotropic [4] and unique [2, 5]. We can further assume that all forms of the matter in the universe are contained in clusters. Each cluster contains sub-clusters and so on. We treat these clusters, at their corresponding scales, as particles of ideal gas. We confine ourself to nonrelativistic regime.

We can represent the universe by a set $G^{n}[2]$

$$
G^{n}=\left\{G_{1}^{n-1}, G_{2}^{n-1}, \ldots, G_{N_{n}}^{n-1}\right\}
$$

where $G_{i}^{n-1}$ represents the $i^{\text {th }}$ cluster and $N_{n}$ is the number of clusters in the $G^{n}$. We can regard that $N_{n}$ is the cardinal number of set $G^{n}$. Similarly we can write $G_{i}^{n-1}$ as

$$
G_{i}^{n-1}=\left\{G_{1}^{n-2}, G_{2}^{n-2}, \ldots, G_{N_{n-1}}^{n-2}\right\}
$$

We can continue this to sub-atomic level and finally we get

$$
G_{j}^{1}=\left\{G_{1}^{0}, G_{2}^{0}, \ldots, G_{N_{1}}^{0}\right\}
$$

where $G_{k}^{0}$ 's correspond to constituent particles which we assume, have no further substructure. 
The spatial distribution of accessible states for this system can be written as $[1,2,6]$

$$
\Gamma_{n}^{\alpha}=\left(\frac{V_{n}^{\alpha}}{\Delta V_{n-1}^{\alpha}}\right)^{N_{n}},
$$

where $\alpha$ stands for configurations space. $V_{n}^{\alpha}$ is the spatial volume of $G^{n}$ and $\Delta V_{n-1}^{\alpha}$ is the volume occupied by $G_{i}^{n-1}$. We assume that $\Delta V_{n-1}^{\alpha}$ is not arbitrarily small. It is much smaller as compared to $V_{n}^{\alpha}$ at the scale of $G^{n}$ whereas at the scale of $G_{i}^{n-1}$, it is much larger than $\Delta V_{n-2}^{\alpha}$. Therefore the corresponding accessible states at the scale of $G_{i}^{n-1}$

$$
\Gamma_{n-1}^{\alpha}=\left(\frac{\Delta V_{n-1}^{\alpha}}{\Delta V_{n-2}^{\alpha}}\right)^{N_{n-1}},
$$

where $\Delta V_{n-2}^{\alpha}$ is the volume occupied by $G_{j}^{n-2}$. Putting $\Delta V_{n-1}^{\alpha}$ from eq.(2) into eq.(1) and on re-arranging we get

$$
\frac{V_{n}^{\alpha}}{\Delta V_{n-2}^{\alpha}}=\left(\Gamma_{n}^{\alpha}\right)^{1 / N_{n}}\left(\Gamma_{n-1}^{\alpha}\right)^{1 / N_{n-1}}
$$

We can generalize it as follow

$$
\frac{V_{n}^{\alpha}}{\Delta V_{0}^{\alpha}}=\prod_{i=1}^{n}\left(\Gamma_{i}^{\alpha}\right)^{1 / N_{i}}
$$

As we know that

$$
s=k_{B} \ln \Gamma,
$$

where $s$ is the entropy and $k_{B}$ is the Boltzmann's constant. Using eq. (4) and eq. (5), we get

$$
\ln \left(\frac{V_{n}^{\alpha}}{\Delta V_{0}^{\alpha}}\right)=\sum_{i=1}^{n} \frac{s_{i}^{\alpha}}{k_{B} N_{i}}
$$

where $s^{\alpha}$ is the spatial entropy and $\Delta V_{0}^{\alpha}$ is the spread or uncertainty in volume of the constituent particles.

It is important to note that a relation describing average value of physical quantities such as entropy, mass or thermal energy of an element of $G^{i}$ with an element of $G^{i-1}$ can be written as

$$
x_{i}=N_{i} x_{i-1}
$$

where $x$ can be the average value of entropy, mass or thermal energy etc. Using eq.(7) for entropy into eq.(6) and after iteration we get

$$
\ln \left(\frac{V_{n}^{\alpha}}{\Delta V_{0}^{\alpha}}\right)=\frac{\mathcal{N} s_{0}^{\alpha}}{k_{B}}
$$


where $s_{0}^{\alpha}$ is the average of spatial entropy of the constituent particle and

$$
\mathcal{N}=1+N_{1}+N_{1} N_{2}+\ldots+\prod_{i=1}^{n-1} N_{i}
$$

It is worth mentioning the last term in the series of $\mathcal{N}$ is much large as compared to other terms in the series.

It follows from eq. (7)

$$
X \equiv x_{n}=\prod_{i=1}^{n} N_{i} x_{0}=N_{t} x_{0}
$$

where $X$ corresponds to the average value of entropy, mass, thermal energy or any other additive physical quantity of the universe $G^{n}$. $N_{t}$ stands for total number of massive particles in the universe. In the above equation, we have further assumed that each $G^{i}$ have equal number of $G^{i-1}$ elements.

If we treat $X$ as entropy in eq. (10) and using this equation and eq.(8), we can obtain the spatial entropy of the universe which can be written as,

$$
S^{\alpha}=\frac{k_{B} N_{t}}{\mathcal{N}} \ln \left(\frac{V_{n}^{\alpha}}{\Delta V_{0}^{\alpha}}\right)
$$

Next we consider momentum space. The momentum distribution of accessible states for $G^{n}$ can be written as [1]

$$
\Gamma_{n}^{\beta}=\frac{V_{n}^{\beta}}{\Delta V_{n-1}^{\beta}}
$$

Here $[1,7]$

$$
V_{n}^{\beta}=\frac{\left(2 \pi m_{n-1} u_{n}\right)^{3 N_{n} / 2}}{\left(3 N_{n} / 2\right) !}
$$

where superscript $\beta$ stands for momentum space. $m_{n-1}$ is the average mass of $G_{i}^{n-1}$ and $u_{n}$ is the average kinetic energy of $G^{n}$.

Similarly we can write

$$
\Gamma_{n-1}^{\beta}=\frac{\Delta V_{n-1}^{\beta}}{\Delta V_{n-2}^{\beta}}
$$

Following the same procedure as for eq. (4), we can write

$$
\prod_{i=1}^{n} \Gamma_{i}^{\beta}=\frac{V_{n}^{\beta}}{N_{1} ! \Delta V_{0}^{\beta}},
$$


where $\Delta V_{0}^{\beta}=\left(\Delta p_{x}\right)^{3 N_{1}}$ and $\Delta p_{x}$ is the uncertainty in momentum of the constituent particle in one dimension. As we reach smaller and smaller $G$ 's, then we are ultimately in quantum regime (i.e. nucleons in a star). A factor of $N_{1}$ ! appears in the denominator of r.h.s of eq. (15) in order to avoid over counting of $N_{1}$ momenta of identical nucleons in stars [1].

After doing some straight forward calculation we can write an expression for entropy due to momentum distribution as

$$
S^{\beta}=\frac{N_{t} k_{B}}{\mathcal{N}+N_{t}}\left\{\ln V_{n}^{\beta}-N_{1} \ln N_{1}+N_{1}-3 N_{1} \ln \Delta p_{x}\right\}
$$

For large $N_{n}[7]$

$$
\ln V_{n}^{\beta} \simeq \frac{3 N_{n}}{2} \ln \left(\frac{2 \pi e}{3 N_{n}} \cdot 2 m_{n-1} u_{n}\right)
$$

where $e=2.718 \ldots$ i.e. base of natural logarithm. Using above approximation we can rewrite eq. (16) as

$$
S^{\beta}=\frac{N_{t} k_{B}}{\mathcal{N}+N_{t}}\left\{\frac{3 N_{n}}{2} \ln \left(\frac{4 \pi e m_{n-1} u_{n}}{3 N_{n}}\right)-N_{1} \ln N_{1}+N_{1}-3 N_{1} \ln \Delta p_{x}\right\}
$$

Now using eqs. (11) and (18), we can write the combined entropy of our universe in phase space as

$$
\begin{aligned}
S & =S^{\alpha}+S^{\beta} \\
& =\frac{N_{t} k_{B}}{\mathcal{N}} \ln \left(\frac{V_{n}^{\alpha}}{\Delta V_{0}^{\alpha}}\right)+\frac{N_{t} k_{B}}{\mathcal{N}+N_{t}}\left\{\frac{3 N_{n}}{2} \ln \left(\frac{4 \pi e m_{n-1} u_{n}}{3 N_{n}}\right)-N_{1} \ln N_{1}+N_{1}-3 N_{1} \ln \Delta p_{x}\right\}(19)
\end{aligned}
$$

As from eqs. (7) and (10), we can write $m_{n-1} N_{n}=m_{n} \equiv M$ and $u_{n} \equiv U$, where $M$ is the mass of the universe, $U$ stands for the total thermal energy of the universe. We can finally write entropy of the universe as

$$
S=\frac{N_{t} k_{B}}{\mathcal{N}} \ln \left(\frac{V}{\Delta V_{0}}\right)+\frac{N_{t} k_{B}}{\mathcal{N}+N_{t}}\left\{\frac{3 N_{n}}{2} \ln \left(\frac{4 \pi e M U}{3 N_{n}^{2}}\right)-N_{1} \ln N_{1}+N_{1}-3 N_{1} \ln \Delta p_{x}\right\},
$$

Here $N_{n}$ is the number of top most clusters (say the number of superclusters in the universe) which makes the universe and $N_{1}$ is the average number of particles in the bottom most cluster (say the number of nucleons in a typical star). In the above equation we have also dropped the indices $\alpha$ and $n$ over the spatial volume of the universe for brevity.

\section{Implication of the Model}

We can now find the thermal energy and the equation of state by using the following thermodynamics relations.

$$
T=\left(\frac{\partial U}{\partial S}\right)_{V, N}
$$


and

$$
\frac{P}{T}=\left(\frac{\partial S}{\partial V}\right)_{U, N}
$$

where $P$ is pressure and $T$ is temperature. From eqs. (20) and (21), we get

$$
U=\frac{3 N_{n}}{2} \cdot \frac{N_{t}}{\mathcal{N}+N_{t}} k_{B} T
$$

This equation gives us the thermal energy of our structureful universe. From eqs. (20) and (22)

$$
P V=\frac{N_{t} k_{B} T}{\mathcal{N}}
$$

Eqs. (23) and (24) can be treated as generalized equations for an ideal gas for the structureful universe.

To verify validity of our system of equations, the ideal gas equations for molecules/particles must be deduced from eqs. (23) and (24). These equations can be obtained, if we take $n=1$ as a special case. In this case $N_{n}=N_{t}$, where $N_{t}$ is the number of particles in a gas. Further we find that for such a system $\mathcal{N}=1$, which can be neglected as compared to $N_{t}$ in the denominator of r.h.s. of eq. (23). We finally get

$$
U=\frac{3}{2} N_{t} k_{B} T
$$

and

$$
P V=N_{t} k_{B} T
$$

The last two equations give us the thermal energy and the equation of state for an ideal gas respectively.

We can get interesting results from these equations. Let us denote the thermal energy (given in eq. (23) of the structureful universe by $U^{A}$ and the pressure by $P^{A}$ (given in eq. (24), and denoting the thermal energy and pressure for structureless universe (given in eqs. (25) and (26) by $U^{B}$ and $P^{B}$ respectively. For same temperature and volume

$$
\begin{gathered}
\frac{U^{A}}{U^{B}}=\frac{N_{n}}{\mathcal{N}+N_{T}} \\
\frac{P^{A}}{P^{B}}=\frac{1}{\mathcal{N}}
\end{gathered}
$$

As we can see that $U^{A} \ll U^{B}$, and $P^{A} \ll P^{B}$. It suggests that expansion of structureful universe can be much slower as compared to a structureless universe. 


\section{Conclusion}

We have considered different scales in expansion of universe starting from sub-atomic scale to super-clusters and beyond. We have obtained generalized ideal gas equations that are applicable to our structureful dynamical universe. It can be applied to stars, galaxies, cluster, supercluster and of course to the universe as a whole. Our model is rather independent of the shape and size of the intermediate clusters. In these derivations we were confined to a non-interacting and nonrelativistic system. Using these results and astrophysical data one can quantitatively compute entropy and other thermodynamical observables of the structureful universe.

\section{Acknowledgments}

We have benefited from useful comments by Ariel Caticha. One of us (SNA) would like to acknowledge the ICSC- World Laboratory for providing partial financial support.

\section{References}

[1] J. C. Lee, Thermal Physics: Entropy and Free Energies, World Scientific, Singapore, 2002.

[2] S.N. Afridi and M.K. Khan, Concepts of Physics, 3, 71, 2006; physics/0412059.

[3] N.A. Bahcall, Clusters and Superclusters of Galaxies, Rep. No. Princeton Observatory Preprint 692; astro-ph/9611148.

[4] C.W. Misner, K.S. Thorne and J.A. Wheeler, Gravitation, W.H. Freeman and Company, New York, 1973.

[5] M. Castagnino, L. Lara and O. Lombardi, Class. Quant. Grav. 20, 369, 2003; quant$\mathrm{ph} / 0211162$.

[6] R. Bowley and M. Sanchez, Introductory Statistical Mechanics, Clarendon Press, Oxford,1996.

[7] F.J. Vesely, Web Tutorial on Statistical Mechanics; http://homepage.univie.ac.at/franz.vesely/sp_english/.

(c)2006 by MDPI (http://www.mdpi.org). Reproduction for noncommercial purposes permitted. 